P141 (continued)

Objective: Evaluation of a classroom-based, teacher-facilitated, nutrition intervention aimed to improve healthy behaviors and knowledge about healthy eating.

Theory, Prior Research, Rationale: Social-ecological model based community-based program designed to be lower-cost, high reach, and align with Common Core standards.

Description: Target audience was students 7-14 years of age $(n=367)$ during school year 2015-2016 in under-served schools in Chicago and Miami. Common Threads' Small Bites community-based program teaches students nutrition through eight 1-hour lessons combined with handson snack preparation. Teachers were trained to deliver Small Bites and received grade-appropriate curriculum to teach in their classrooms.

Evaluation: Small Bites effects were assessed using a quasi-experimental, pre-post survey design. The majority of participants qualified for free- or reduced-price lunch (84\%). Binary and count variables were assessed with generalized linear mixed models, ordinal variables with cumulative logistic mixed models. Participation in Small Bites resulted in a net increase in students with improved nutrition knowledge $(32 \%, \mathrm{p}<.001)$ and higher odds of answering individual questions correctly (all $\mathrm{p}<.001$ ). Net number of students who consumed a greater variety of colored vegetable groups at least once the previous day increased after programing $(9 \%, \mathrm{P}=0.03)$ and amount consumed of each vegetable color group increased for two out of four vegetable groups $(\mathrm{P} \leq .001)$. The odds of a student reporting agreement with the statement "I show my family how to cook at home" increased after programing $(\mathrm{OR}=1.47, \mathrm{P}=.05)$.

Conclusions and Implications: Small Bites increased nutrition knowledge, vegetable intake, and healthy behaviors, supporting nutrition education as an important step in healthy behavioral changes.

Funding: Walmart Foundation; Whole Kids Foundation

\section{P142 The Challenge of Increasing Fruit and Vegetable Consumption Among Jamaican Adults}

Vanessa White-Barrow, MS, BS, vanowhite@gmail.com, University of Technology, Jamaica, 237 Old Hope Road, Kingston 6, Kingston, Jamaica; Kerry Weatherly, MS, RD, University of Technology, Jamaica; Novelette MattisRobinson, MPH; Renelle Aarons, MS; Fitzroy Henry, PhD

Objective: Compare the Transtheoretical Model (TTM) stages of change (SOC) for fruit and vegetable consumption among Jamaican adults and evaluate the dominant factors influencing each SOC.

Study Design, Setting, Participants: A household based cross-sectional survey among 1,057 adults 18 - 69 years selected from five parishes in Jamaica, was conducted using an interviewer administered questionnaire over a five month period.

Outcome Measures and Analysis: Socio-demographic, height, weight, fruit and vegetable consumption SOC, self- efficacy, positive perceptions, barriers and social support data; were collected. Overweight and obesity were defined based on BMI while fruit and vegetable consumption SOC were scored then analysed using Chi-square, analysis of variance and regression.

Results: Fruit and vegetable consumption of few respondents $(20.5 \%)$ were in the action/maintenance SOC and significantly more females were in contemplation/preparation $(\chi=12.98 ; p=0.01)$. No significant differences were observed in SOC for fruit and vegetable consumption by BMI status. A significant increase in fruit and vegetable consumption self-efficacy scores was detected from pre/ contemplation to action/maintenance $(\mathrm{F}=51.13, \mathrm{p}<$ .001). Significant differences were seen in mean scores for the perceived benefits of fruits and vegetables by SOC $(\mathrm{F}=23.22, \mathrm{p}<.001)$. Adults in pre-contemplation were more likely to perceive barriers than those in the action/ maintenance SOC $(\mathrm{F}=20.03, \mathrm{p}<0.001)$; and minimal support from family (mean score 10.59 of 21 ) and friends (8.57 of 21) was reported.

Conclusions and Implications: Females are least likely to start or maintain participation in programmes designed to increase fruit and vegetable consumption. Community based interventions designed to build self-efficacy and involve social support systems may successfully address this.

Funding: University of Technology, Jamaica Research Development Fund

\section{P143 The Effect of Low Sodium Policy in Congregate Meal Program: A Pilot Study}

Hee-Jung Song,PhD, hjsong@umd.edu, University of Maryland College Park, 0112 Skinner Building, Department of Nutrition and Food Science, College Park, MD 20742; La Tayna D. Clark, MS, LDN, RD, Anne Arundel County, Maryland; Amanda Ashley, University of Maryland College Park; Gwyneth Bradshaw; Allie Hosmer; Penelope Taylor

Objective: Since 2014, the Older American Act Nutrition Program (OAANP) in Maryland has implemented a low-sodium policy for congregate meals, but no evaluation has been conducted. This study presents the findings of the low-sodium policy and discusses a possible senior centerbased hypertension program while supporting the intent of the policy.

Study Design, Setting, Participants: Using pre, poststudy design, data were collected from hypertensive older adults who consumed congregate meals on a regular basis $(n=67)$ before and after policy implementation. Paired $t$ test and Wilcoxon signed ranks test were used for data analysis.

Outcome Measures and Analysis: The Block sodium screener and a structured questionnaire and were used to estimate dietary sodium intake and other hypertension risk factors including BMI, self-care behaviors, medication taking, and hypertension knowledge. 
P143 (continued)

Results: A year after OAANP's low-sodium policy, average sodium intake was statistically significantly reduced $(\mathrm{p}<.001), 2329.7 \pm 177 ; 1021.9 \mathrm{mg}$ and $1959.7 \pm 177$; 1026.2 at pre- and post-survey respectively. Also, the proportion of older adults consuming $\geq 2,300 \mathrm{mg}$ sodium was also significantly reduced $(\mathrm{p}=.002)$. At baseline, $97.5 \%$ participants took anti-hypertensive medication and about $40 \%$ participants still had uncontrolled blood pressure $(\geq 150 / 90)$. A majority of participants $(82.9 \%)$ were overweight or obese. Respectively, 68.4 and $65.4 \%$ ate less than 3-4 servings of vegetables and 2-3 servings of fruit. As expected, these key hypertension risk factors haven't been changed at post survey. Also, average blood pressure tended to increase at postsurvey, although it was not statistically significant $(\mathrm{p}=.06)$.

Conclusions and Implications: Utilizing a congregate meal program to reduce sodium intake for older adults is an effective strategy. To address hypertension related risk factors, however, further multifaceted intervention strategies are necessary.

Funding: None

\section{P144 The Impact of a Gleaning Program on Vegetable Intake in Low-Income Seniors}

Deborah Lown, $P h D, R D$, Grand Valley State University; Lisa G. Sisson, MM, RD, sissonl@gvsu.edu, Grand Valley State University, 401 Fulton Street West, Grand Rapids, MI 49504

Objective: The purpose of this project was to determine if a gleaning program improved vegetable intake in low-income seniors receiving food from The Emergency Food Assistance Program (TEFAP).

Study Design, Setting, Participants: Low-income individuals have difficulty accessing healthy foods for a variety of reasons, including access and cost. To increase access, fresh produce gleaned from growers at a farmers market was dispensed with the monthly TEFAP distribution at 5 sites in Ottawa County, Michigan. Seniors receiving TEFAP foods were allowed to make selections from the produce and were asked to complete a survey on their vegetable intake before and after this new gleaning program.

Outcome Measures and Analysis: Descriptive statistics were used to describe seniors participating in TEFAP at the 5 study sites. One-sample t-test was used to evaluate change in vegetable intake.

Results: The thirty-six clients who completed the pre-survey in June and the post survey in September were mainly Caucasian females who lived alone with a mean age of 70.5 years. Only half of those surveyed participated in Supplemental Nutrition Assistance Program (SNAP) and fewer participated in other available food assistance programs. The mean monthly intake of vegetables significantly increased from 11 to 22 servings per month $(<0.0001)$.
Conclusions and Implications: This study indicates the gleaning program was successful because it significantly increased the intake of vegetables among low-income seniors. Unfortunately, these clients were not utilizing all of the available food assistance programs to improve access to healthy foods. Additional education on SNAP and other food assistance programs is indicated to assist with cost, access and intake of fruits and vegetables.

Funding: None

\section{P145 The Snack Zone: Development of a Community-Based Snacking Program for Individuals with Autism}

Janice Goldschmidt, MS, LDN, RD, jgoldschmidt@css-md. org, Community Support Services, Inc., 9075 Comprint Court, Gaithersburg, MD 20877

Objective: To describe an effective community-based snacking program for adults with autism and other developmental disabilities and elucidate the rationale behind the design and implementation.

Theory, Prior Research, Rationale: At present there is no formal definition of what constitutes appropriate snacking behavior including the appropriate number of calories. This presentation makes the case for snacking through choice of unlimited nutrient dense foods accompanied by limited amounts of energy dense toppings. Total energy consumption in "The Snack Zone" is aimed at approximately 200 high quality calories per visit.

Description: Although underrepresented in most national dietary surveys, secondary studies of individuals with autism strongly suggest high rates of both overweight/obesity and the chronic conditions that are typically comorbid. Despite these trends, few nutrition programs have been developed for this at-risk population. "The Snack Zone" is a community based snacking program designed for individuals with autism, though the principles are applicable to other populations. Visits to "The Snack Zone" are based on the premise that, if an individual is hungry, the menu options will guide them to an appropriate high quality snack. Over time, the principles supported in the program can be generalized to reinforce healthy eating patterns.

Evaluation: Attendance/consumables were tracked for one week. Along with energy intake analysis, 200 individuals were found to have snacked on carrots (91 servings), tomatoes (47), celery (59), cucumber (52), apples (97), and oranges (90).

Conclusions and Implications: To avoid adversely impacting energy balance leading to obesity, appropriate snacking needs to be defined and supported. "The Snack Zone" accomplishes this goal and is creatively implemented such that participants can pick and choose based on individual preferences.

Funding: None 